\title{
The Influence of Plasma Plume in Laser Milling for Mold Manufacturing
}

\author{
Giovanni TANI ${ }^{*}$, Leonardo ORAZI ${ }^{* *}$, Alessandro FORTUNATO* ${ }^{*}$, and Gabriele CUCCOLINI ${ }^{* *}$ \\ *DIEM Department of Mechanical Construction Engineering, University of Bologna, Viale Risorgi- \\ mento, 2 40136, Bologna, Italy. \\ E-mail: giovanni.tani2@unibo.it \\ ** DISMI Department of Science and Method for Engineering, University of Modena-Reggio Emilia, \\ Via Amendola, 2 42100, Reggio Emilia, Italy.
}

\begin{abstract}
The paper refers to the modeling of the plasma plume influence on the shape of the crater obtained by means of nanosecond pulsed laser milling. A transient model of the physical state of the plasma plume is developed according to the laser parameters. Two empirical coefficients are proposed in the model in order to evaluate the plasma plume self-emission energy lost towards the environment and the energy spread from the plasma towards the target surface.

These two coefficients, directly correlated to the depth and to the width of the crater, can be experimentally determined, due to the difficulty of their analytical quantification, and they can be used for tuning a complete plasma plume software package for laser milling simulation named LAS (Laser Ablation Simulator) already developed by the authors.

In this paper their influence on the crater shape will be proved by means of several simulation runs.
\end{abstract}

Keywords: Laser milling, FDM, process planning, plasma plume modeling, mesh

\section{Introduction}

Laser milling is a manufacturing technology characterized by the possibility of creating small and complex shapes with good accuracy even in hardened alloys. The short working times, if compared to those concerning EDM, and the possibility of integrating the laser source directly on the manufacturing system, determined the great interest of today's industry towards this technology. The great number of parameters involved in the process makes optimization quite difficult and time expensive especially if performed through a trial-and-error technique. According to this the possibility of exploiting a reliable and accurate numerical model in order to simulate the process is a time and cost-effective topic.

Several scientific works have been proposed in the last years for modelling the physical phenomena occurring during the laser material interaction in laser ablation with long pulse width [1][2][3][4][5][6][7][8]. These interactions typically are: the energy absorption of the workpiece, the energy transfer to the lattice, the phase transformations occurring in the target material, the ablation mechanisms, the properties of the induced plasma and its interaction with the incident laser beam.

The authors already presented a detailed 3D transient numerical model were all the above mentioned aspects were implemented [9]. The basic assumptions for the plasma modelling were adopted according to [5] and [11] and they can be summarized as follows:

- the plasma plume is in local thermodynamic equilibrium (LTE) with homogeneous temperature, pressure, and ion distribution;
- the recession velocity of the ablating surface is evaluated according to the Hertz-Knudsen equation assuming that the explosive effects are negligible and the gas expansion, from the surface target, produces a sonic front;

- the plasma plume is supposed to have cylindrical shape with length depending on the gas expansion velocity. These simplifications make possible to avoid the solution of $\mathrm{Na}$ vier - Stokes equation and the simulation is drastically simplified.

The physical state of the plasma plume determines the amount of laser energy absorbed by the plume itself and, subsequently, the energy delivered to work-piece. Anyway the previous hypotheses are too simplified when a detailed prediction of the ablated crater have to be achieved. This is mainly due to the fact that the energy lost for irradiation from the plume is very approximately quantified, [5] while the energy transferred to target surface is not considered. Those two physical aspects greatly influence the crater shape and an accurate modelling has nowadays not yet been proposed in scientific literature due the high complexity of the involved phenomena.

In [10] the authors proposed an empirical coefficient $\eta_{P}$ for the evaluation of the plasma plume self-emission energy lost towards the environment which allows to better evaluate the laser energy reaching the workpiece. This coefficient, as showed in [10], influences the depth of the ablated crater. In that work, no considerations were done about the energy spread from the plume reaching the target surface even if this contribution influences the crater width.

Moreover, the energy amount spread from the plume that impacts the surface workpiece is very difficult to be 
mathematically quantified and no work was presented in literature about this topic.

In this paper a second empirical coefficient $\rho_{P}$ is proposed which has to be experimentally determined according to the workpiece material.

Several simulations will be carried out and the influence of both coefficients $\eta_{P}$ and $\rho_{P}$ on crater shape will be proved. The need of experimental trials for the evaluation of the coefficients will be underlined.

The capability of the laser simulator package (LAS) in laser milling process planning is also shown.

\section{The laser milling modeling}

The process simulator LAS is developed considering both the interactions between the self-induced plasma plume and laser beam and target material and the laser beam emerging from the plume. The explanation is presented in following paragraphs.

\subsection{Plasma plume modeling}

The detailed model for the prediction of the physical state of the plume was already presented in [9][10] and it will be briefly exposed in this article.

The plasma, emerging from the irradiated target surface, is supposed in local thermal equilibrium (LTE) and the relative time dependent equilibrium temperature $T_{P}$ is calculated by solving the following equation system:

$$
\left\{\begin{array}{l}
\frac{d T_{P}}{d t}=\frac{I_{L}\left[1+R_{L} e^{\left.-\left(\frac{\alpha_{a b s} l}{\cos \vartheta}\right)\right] \cdot\left[1-e^{-\left(\frac{\alpha_{a b s} l}{\cos \vartheta}\right)}\right]-\left(1-R_{P}\right) I_{P}}\right.}{k_{B} N l \gamma(Z+1)\left(\frac{M^{2}}{2}+\frac{1}{\gamma(\gamma-1)}\right)} \\
I_{P}=4 \sigma \alpha_{a b s} T_{P}^{4} \\
\frac{d l}{d t}=\sqrt{\frac{\gamma(Z+1) k_{B} T_{P}}{m}} \\
\frac{d(N l)}{d t}=P_{V}\left(T_{S}\right) \frac{1}{\sqrt{2 \pi k_{B} T_{S}}} \\
p_{V}\left(T_{S}\right)=p_{b} e^{\frac{\Delta H \cdot m}{k_{B}}\left(\frac{1}{T_{B}}-\frac{1}{T_{S}}\right)}
\end{array}\right.
$$

where:

$R_{L}$ Reflectivity of laser radiation

$R_{P}$ Reflectivity of self-emission plasma plume radiation

$p_{b} \quad$ Boiling pressure [Pa].

$\mathrm{p}_{V}$ Vaporization pressure [Pa].

$\Delta H$ Heat of vaporisation per atom $[\mathrm{J} / \mathrm{kg}]$

$k_{B}$ Boltzmann constant $[\mathrm{J} / \mathrm{K}]$

$\sigma \quad$ Stefan constant $\left[\left(\mathrm{W} / \mathrm{m}^{2}\right) / \mathrm{K}^{4}\right]$

$\vartheta \quad$ Laser beam slope with respect to the workpiece

$m$ Atomic mass [kg]

$T_{b} \quad$ Boiling temperature $[\mathrm{K}]$

$T_{s} \quad$ Surface temperature $[\mathrm{K}]$
$T_{p} \quad$ Plasma plume temperature $[\mathrm{K}]$

$I_{L}$ Intensity of the incident laser radiation $\left[\mathrm{W} / \mathrm{m}^{2}\right]$

$l \quad$ Plasma plume length [m]

$N$ Average atom+ion density $\left[1 / \mathrm{m}^{2}\right]$

$\gamma \quad$ Ratio of the specifics heats

$Z$ Charge state of the ion

$M$ Mach number

$I_{P} \quad$ Plasma plume self-emission energy $\left[\mathrm{W} / \mathrm{m}^{3}\right]$

$\alpha_{a b s}$ Plasma absorption coefficient $[1 / \mathrm{m}]$

The intensity of the incident laser radiation $I$, passing through the plume with an absorbing coefficient $\alpha_{a b s}$ evaluated in equation (3), is calculated by means of equation 2 :

$$
I(x, y, z)=I_{L} \cdot e^{\left(-\frac{\alpha_{a b s} \cdot l}{\cos \vartheta}\right)}
$$

The model refers only to the plasma ionization and to the plasma heating phases while the recombination phase, corresponding to the plasma cooling, is not investigated.

According to [5] the radiation absorption due to the inverse of bremsstrahlung $(I B)$ is not sufficient to sustain the plasma formation and for this reason the photoionization (IP) of the excited state must be considered for the vapour breakdown [11]. Moreover, considering that the ionization process is described by the Saha law, the coefficient of the full laser radiation absorption is:

$$
\begin{gathered}
\alpha_{a b s}=\alpha_{I B}+\alpha_{I P} \\
\alpha_{I B}=3.69 \cdot 10^{8} \frac{Z^{3} N_{i}^{2}}{v^{3} \sqrt{T_{P}}}\left[1-e^{\left(\frac{h v}{k_{B} T_{P}}\right)}\right] \\
\alpha_{P I}=\sum_{n}\left(7.9 \cdot 10^{18} N\left(\frac{E_{n}}{h v}\right)^{3} \sqrt{\frac{I}{E_{n}}}\right)
\end{gathered}
$$

where:

$N_{i} \quad$ Number of ions

$\checkmark \quad$ Laser radiation frequency $[\mathrm{Hz}]$

$E_{n}$ Activation energy [J]

$h$ Planck constant [Js]

The power intensity reaching the workpiece $I_{W b}$, is calculated by means of equation 6 while the power balance in the plasma plume, $I_{P b}$, is written in equation 7 .

$$
\begin{aligned}
& I_{W b}=\left(1-R_{P}\right)\left(1-\eta_{P}\right) I_{P}+\left(1-R_{L}\right) \lambda_{P} I_{L} \\
& I_{P b}=I_{L}\left(1-\lambda_{P}\right)\left(1+R_{L} \lambda_{P}\right)-I_{P}\left[1-R_{P}\left(1-\eta_{p}\right)\left(1-\lambda_{p}\right)\right]
\end{aligned}
$$

where:

$$
\lambda_{P}=e^{-\alpha_{a b s} l}
$$

and $\eta_{p}$ is a coefficient to be tuned. 
The explanation of the previous equations is figure 1 where the yellow arrows represent the intensities of the laser beam while cyan arrows are the emitted intensities.

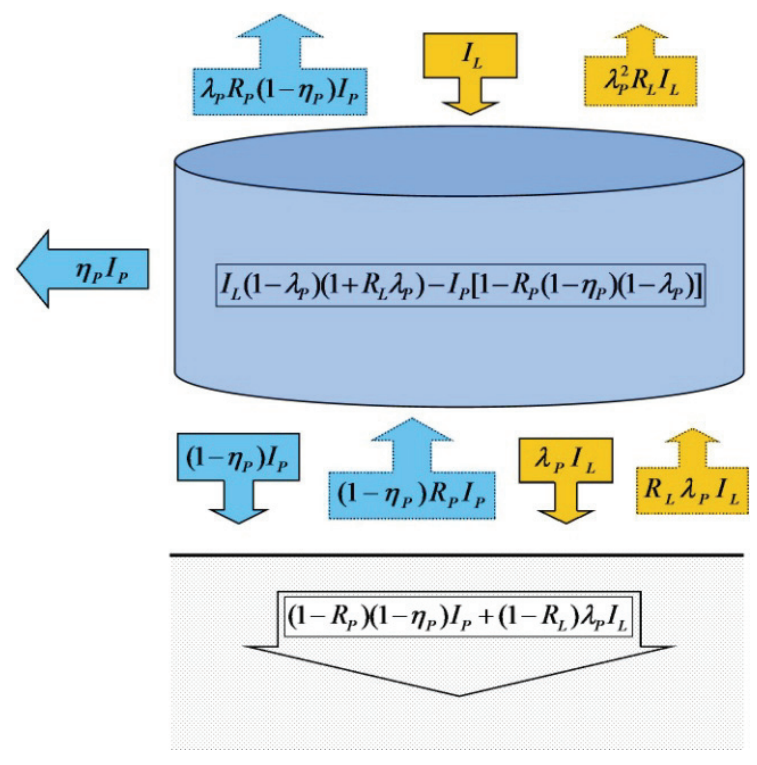

Fig. 1 The intensity balance of the plasma plume and of the workpiece.

\subsection{The ablation modeling}

The numerical modeling of laser milling with nanosecond pulse width laser [9] is obtained by solving the Fourier equation for the heat flux into the workpiece, see equation 9 , and by solving equation 10 for the surface recession velocity of the liquid-vapour interface when the normal vaporization model is adopted [3]:

$$
\begin{gathered}
c_{p} \rho \frac{\partial T}{\partial t}=\frac{\partial}{\partial x}\left(k \frac{\partial T}{d x}\right)+\frac{\partial}{\partial y}\left(k \frac{\partial}{\partial y}\right)+\frac{\partial}{\partial z}\left(k \frac{\partial}{\partial z}\right)+q \\
\left(\frac{\partial z}{\partial t}\right)_{z \approx 0} \approx p_{v}\left(T_{S}\right) \frac{1}{\sqrt{2 \pi m k_{B} T_{S}}}\left(\frac{m}{\rho}\right)
\end{gathered}
$$

where:

$C_{p} \quad$ Target specific heat $[\mathrm{J} / \mathrm{kgK}]$

$\rho$ Target density $\left[\mathrm{kg} / \mathrm{m}^{3}\right]$

$q$ Heat generation in the generic $(\mathrm{x}, \mathrm{y}, \mathrm{z})$ point $\left[\mathrm{W} / \mathrm{m}^{3}\right]$

$T$ Target temperature [K]

$k$ Target thermal conductivity [W/mK]

The numerical solution of the equations is obtained by means of FDM method which represents the best solution for the study of no stationary phenomena with variable boundary condition like in pulsed laser applications with moving spot.

Figure 2 reports the physical concepts adopted for the modelling of the laser-material interaction and plasma plume expansion.

\section{Results and discussion}

As pointed out in [10] the plasma plume determines the energy delivered to the workpiece and so it influences the shapes of the crater. In particular according to the power density lost for irradiation and the to the plume energy spread towards the target surface the crater depth and width can be sensibly modified.

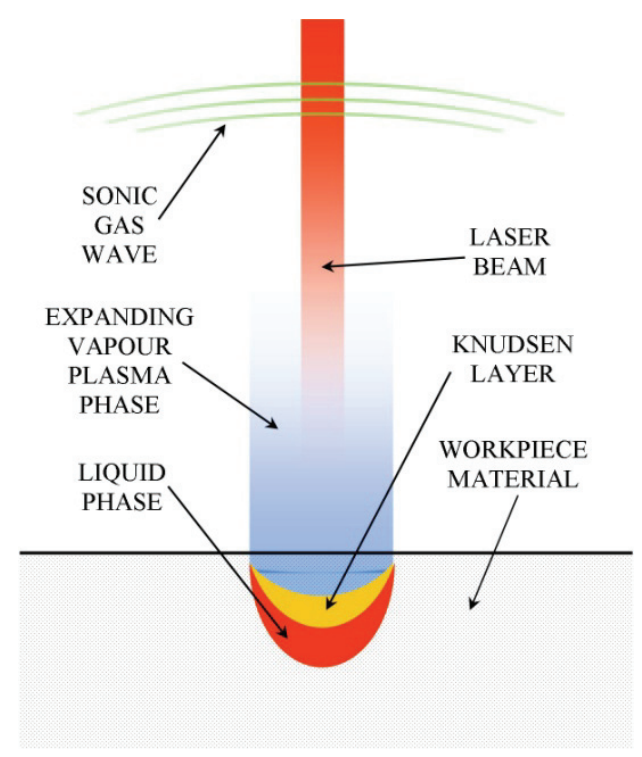

Fig. 2 The laser-material interaction and the plasma plume expansion.

In order to take into account these effects, which are very difficult to be mathematically quantified, in this paper two empirical coefficients are proposed, $\eta_{P}$ and $\rho_{P}$, which have to be experimentally tuned according to the workpiece material. Coefficient $\eta_{P}$ is the ratio between the emitted intensity lost in the environment and the emitted intensity of the plume while coefficient $\rho_{P}$ quantifies the radius of irradiation of the plume. Increasing $\rho P$ the energy for unit surface decrease. By varying them, the predicted crater assumes the shape showed in figures $3,4,5,6$.

In figures 3 and 4 the predicted craters obtained on a steel workpiece material when coefficient $\rho_{P}$ is varied, are shown. The simulation was set in order to obtain three adjacent craters maintaining the laser parameters constant for all the craters and equal to: $I_{L}=1.2 \cdot 10^{13} \mathrm{~W} / \mathrm{cm}^{2}, T E M_{00}$ a spot diameter $d=20 \mu \mathrm{m}$ and a laser frequency of $1 \mathrm{kHz}$. The laser source is a Nd:Yag with a wave length of $1064 \mathrm{~nm}$.

If $\rho_{P}=10$ the predicted craters are represented in figure 4 . Coefficient $\eta_{P}$ is still equal to 0.5 which means that the $50 \%$ of the plasma plume energy is dispersed towards the environment from the plasma plume. 


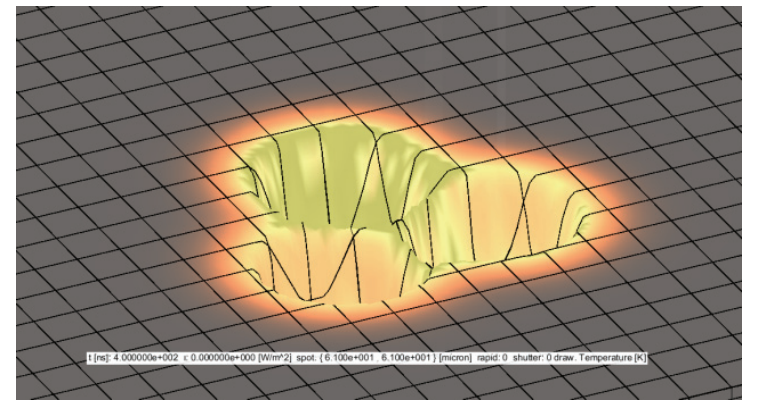

Fig. 3 Influence of coefficient $\rho_{P}$ on the crater depth on steel material: $\eta_{P}=0.5$ and $\rho_{P}=0$. The mesh dimension is: $\mathrm{dx}=\mathrm{dy}=10 \mu \mathrm{m}, \mathrm{dz}=2 \mu \mathrm{m}$.

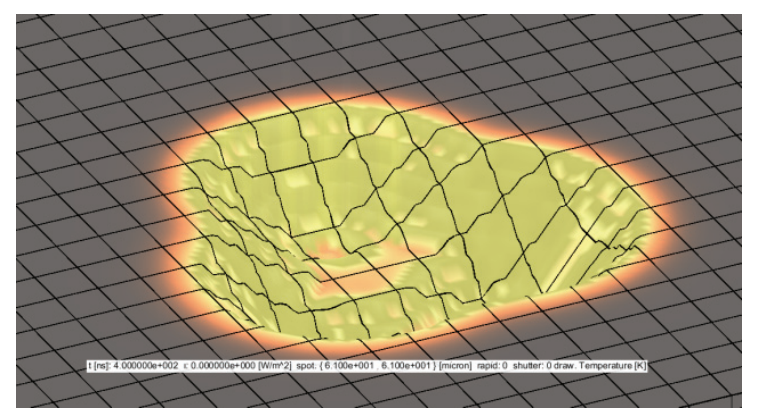

Fig. 4 Influence of coefficient $\rho_{P}$ on the crater depth on steel material: $\eta_{P}=0.5$ and $\rho_{P}=10$. The mesh dimension is: $\mathrm{dx}=\mathrm{dy}=10 \mu \mathrm{m}, \mathrm{dz}=2 \mu \mathrm{m}$.

By comparing the figures, it is evident that increasing $\rho_{P}$ the crater depth decreases with respect to $\rho_{P}=0$ while the crater width becomes larger. In both figures 3 and 4 the simulations time was stopped after $400 \mathrm{~ns}$. In figures 5 and 6 the plasma plume is also represented on the craters.

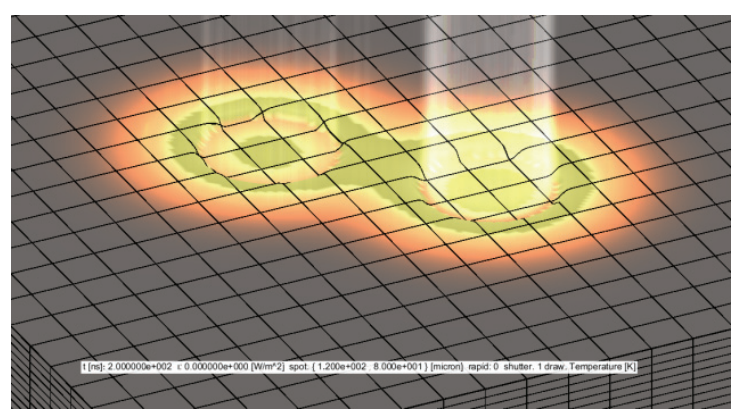

Fig. 5 Influence of coefficient $\eta_{P}$ on the crater depth on steel material: $\eta_{P}=0.8$ and $\rho_{P}=0$. The mesh dimension is: $\mathrm{dx}=\mathrm{dy}=10 \mu \mathrm{m}, \mathrm{dz}=2 \mu \mathrm{m}$.

Two simulations are presented in figures 5 and 6 in order to show the influence of $\eta_{P}$ coefficient on the crater depth. In particular, in figure $5 \eta_{P}=0.8$ and in figure 6 $\eta_{P}=0.5$ and $\rho_{P}=0$ for both the simulations which means that no contribution to the crater irradiation comes from the energy irradiated from the plume. The laser parameters are $I_{L}=0.5 \cdot 10^{13} \mathrm{~W} / \mathrm{cm}^{2}, T E M_{00}$ a spot diameter $d=40 \mu \mathrm{m}$. The simulation time was stopped after $200 \mathrm{~ns}$ and the predicted crater depths are, respectively, $2.02 \mu \mathrm{m}$ and $39.8 \mu \mathrm{m}$.

Analyzing figures 3, 4, 5, and 6 it is evident the great influence of the proposed coefficients $\eta_{P}$ and $\rho_{P}$ on the crater shapes while, at the same time, they are very difficult to be analytically quantified. For these reasons in order to predict a crater shape as accurate as possible, they can be varied to tune the predicted shapes to the experimental ones according to the workpiece material.

The experimental trials are now in progress and the results will be published in the future works.

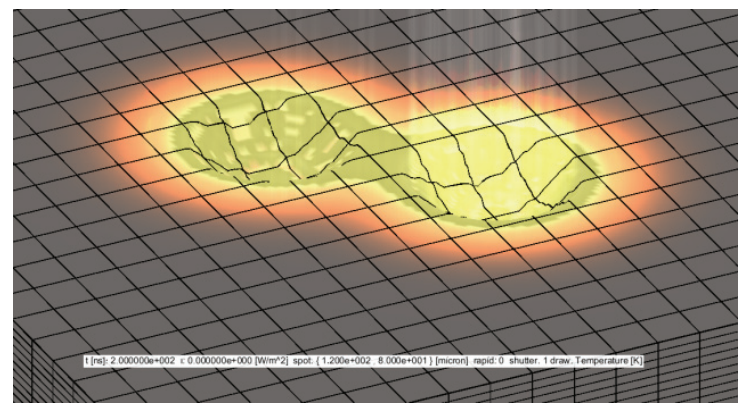

Fig. 6 Influence of coefficient $\eta_{P}$ on the crater depth on steel material: $\eta_{\mathrm{P}}=0.5$ and $\rho_{\mathrm{P}}=0$. The mesh dimension is: $\mathrm{dx}=\mathrm{dy}=10 \mu \mathrm{m}, \mathrm{dz}=2 \mu \mathrm{m}$.

\section{Industrial application}

As said in the previous paragraphs, the plasma plume model is a part of a wider software tool, developed by the authors, dedicated to the laser milling simulation named LAS. LAS is created in order to guarantee the maximum flexibility for the process simulation and for the industrial applications. The graphic outputs were implemented by using OpenGL $\mathrm{T}_{\mathrm{TM}}$ libraries and the ablation process can be visualized during the simulation.

The software implements three classes of controls that act on the spatial and temporal laser intensity distribution independently from each other:

- Laser Source intensity, distribution, frequency, duty cycle...

- Scanning Head Working and "rapid" movements...

- Shutter Simulations of the various galvo delays, pulse suppression, ecc...

The laser beam trajectories can be commanded by means of the ISO code as in the example shown in figures 7, 8 and 9 where the word "LPM" is reproduced on a steel sheet plate. The laser beam parameters are: $I_{L}=1 \cdot 10^{13}$ $\mathrm{W} / \mathrm{cm}^{2}, T E M_{00}$ and spot diameter $d=10 \mu \mathrm{m}$ and a laser frequency of $1 \mathrm{kHz}$.

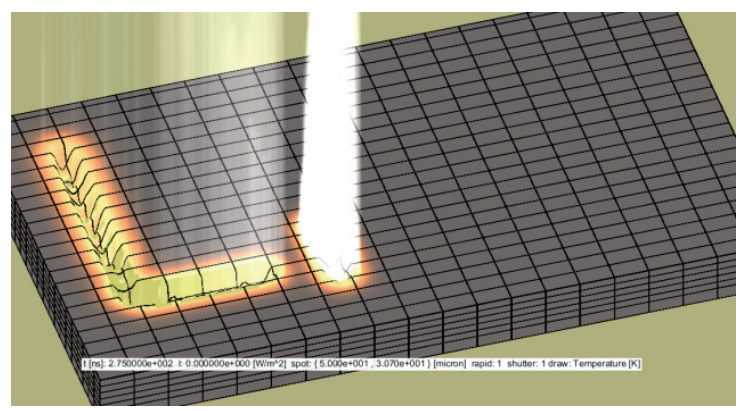

Fig. 7 Micro-milling of the LPM features after 250 ns of simulation. The mesh dimension is: $\mathrm{dx}=10 \mu \mathrm{m} ; \mathrm{dy}=5 \mu \mathrm{m}$, $\mathrm{dz}=2 \mu \mathrm{m}$. 


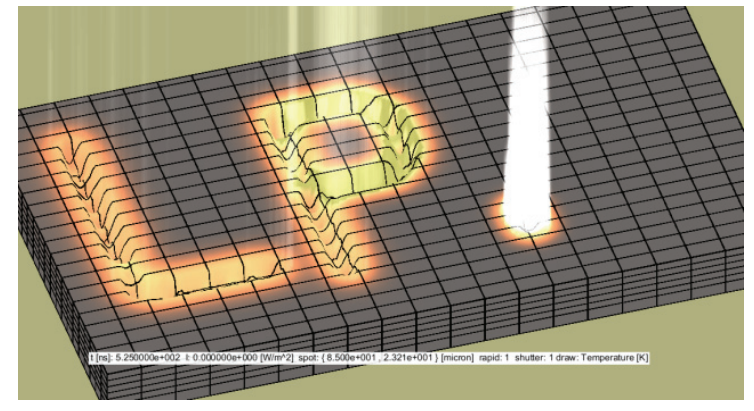

Fig. 8 Micro-milling of the LPM features after 525 ns of simulation. The mesh dimension is: $\mathrm{dx}=10 \mu \mathrm{m} ; \mathrm{dy}=5 \mu \mathrm{m}$, $\mathrm{dz}=2 \mu \mathrm{m}$.

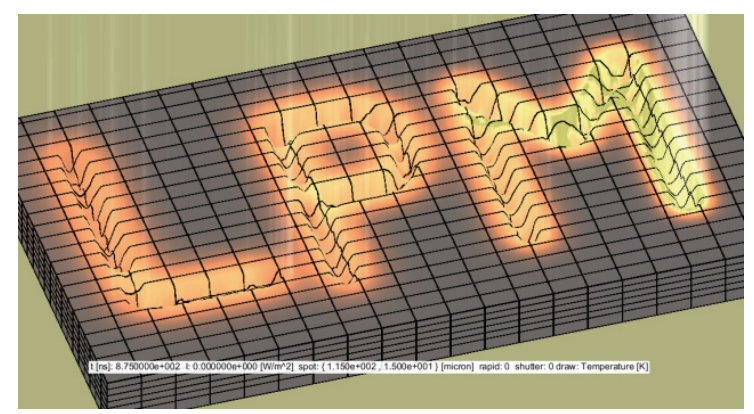

Fig. 9 Micro-milling of the LPM features after 875 ns of simulation. The mesh dimension is: $\mathrm{dx}=10 \mu \mathrm{m} ; \mathrm{dy}=5 \mu \mathrm{m}$, $\mathrm{dz}=2 \mu \mathrm{m}$.

The ISO commands, for the laser beam displacements and for the shutter, used for the previous process simulation are reported in figure 10.

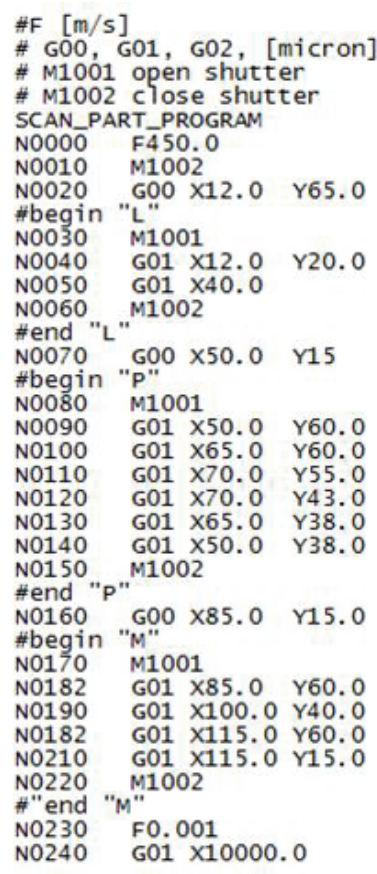

Fig. 10 ISO commands used for LAS input to create the LPM features
Two empirical coefficients for the evaluation of the plasma plume self-emission energy lost towards the environment and for the evaluation of the plasma plume spread on the target surface are proposed.

Several simulations are proposed to underline their influence on the crater shape together with the necessity of their experimental quantification.

The facilities of the Laser Ablation Simulator (LAS) for the laser milling characterization is shown.

\section{Acknowledgments}

The authors would like to thank CB Ferrari S.P.A. for the financing support.

\section{References}

[1] M. Modest: International Journal of Heat and Mass Transfer, 39 (1996) 2. (Journals)

[2] N. Bulgakova, and A. Bulgakov: Applied Physics A, 73 (2001) 2. (Journals)

[3] R. Kelly, and A. Miotello: Applied Surface Science, 96-98 (1996). (Journals)

[4] N. Bulgakova, A. Bulgakov, I. Bourakov, and N. Bulgakova: Applied Surface Science, 197-198 (2002). (Journals)

[5] J. G. Lunney, and R. Jordan: Applied Surface Science, 127-129 (1998). (Journals)

[6] S. R. Franklin, and R. Thareja: Applied Surface Science, 222 (2004) 1-4. (Journals)

[7] T. Dobrev, D. Pham, and S. Dimov: Proc. of $1 \mathrm{st}$ International Conference on Multi-Material Micro Manufacture, (2005). (Conference Proceedings)

[8] A. Bogaerts, Z. Chen, R. Gijbels, and A. Vertes: Spectrochimica Acta Part B, 58 (2003) 11. (Journals)

[9] G. Tani, L. Orazi, A. Fortunato, and G. Cuccolini: Proc. of IMECE2006 2006 ASME International Mechanical Engineering Congress and Exposition, ASME, (2006). (Conference Proceedings)

[10] G. Tani, L. Orazi, A. Fortunato, and G. Cuccolini: Proc. of Photonisc West, SPIE (2007). (Conference Proceedings)

[11] J. Neamtu, I. Mihailescu, C. Ristoscu, and J. Hermann: Journal of Applied Physics, 86 (1999) 11. (Journals)

(Received: April 24, 2007, Accepted: October 11, 2007)

\section{Conclusion}

